\title{
A model of the rise and fall of roads
}

\section{Lei Zhang}

University of Maryland

lei@umd.edu

\section{David Levinson}

University of Minnesota

dlevinson@umn.edu
Abstract: This paper analyzes the relationship between network supply and travel demand and describes a road development and degeneration mechanism microscopically at the link (road-segment) level. A simulation model of transportation network dynamics is developed, involving iterative evolution of travel demand patterns, network revenue policies, cost estimation, and investment rules. The model is applied to a real-world congesting network for Minneapolis-St. Paul, Minnesota (Twin Cities), which comprises nearly 8000 nodes and more than 20,000 links, using network data collected since 1978. Four experiments are carried out with different initial conditions and constraints, the results of which allow us to explore model properties such as computational feasibility, qualitative implications, potential calibration procedures, and predictive value. The hypothesis that road hierarchy is an emergent property of transportation networks is corroborated and the underlying reasons discovered. Spatial distribution of capacity, traffic flow, and congestion in the transportation network is tracked over time. Potential improvements to the model, in particular, and future research directions in transportation network dynamics, in general, are also discussed.

\section{Article history:}

Received: February 4, 2016

Accepted: April 20, 2016

Available online: September 19, 2016

\section{$1 \quad$ Introduction}

Transportation network planning decisions made at one point in time can have profound impacts on the future. However, transportation networks are usually assumed to be static in models of land use. A better understanding of the natural growth pattern of roads would provide valuable guidance to planners. Today, the dynamics of network growth are poorly understood. This lack of understanding is revealed time and again in the long-range planning efforts of metropolitan planning organizations (MPOs), where transportation network change is treated exclusively as the result of top-down decision-making, without considering what drives those decisions (Montes de Oca and Levinson 2006; Yusufzyanova and Zhang 2011a; Zia and Koliba 2015). Non-immediate and non-local effects are generally ignored in planning practice because network effects are not predicted with current tools, which often results in myopic network expansion decisions. If one looks at the complexity and bureaucracy involved in transportation infrastructure investment, one might conclude that it is impossible to model the transportation network dynamics endogenously. However, changes to the transportation network

Copyright 2015 Lei Zhang \& David Levinson

http://dx.doi.org/10.5198/jtlu.2016.887

ISSN: 1938-7849 | Licensed under the Creative Commons Attribution - Noncommercial License 3.0

The Journal of Transport and Land Use is the official journal of the World Society for Transport and Land Use (WSTLUR) and is published and sponsored by the University of Minnesota Center for Transportation Studies. 
are rather the result of numerous small decisions (and some large ones) by property owners, firms, developers, towns, cities, counties, state department of transportation districts, metropolitan planning organizations (MPOs), and states in response to market conditions and policy initiatives (Barthelemy 2015). Though institutions make network growth (decline) happen on the surface, network dynamics are indeed also driven by some underlying natural market forces and hence predictable.

A transportation network is a complex system that exhibits the properties of self-organization and emergence. Much previous research in dynamics related to transportation networks focuses on traffic assignment or traffic management. However, the dynamics of transportation network growth have received limited, though growing, attention. (Xie and Levinson [2009] reviews the literature to 2009.) If a transportation network is represented by a directed graph, there are several important questions not fully answered:

(1) How do the existing links, alternatively called arcs or edges and defined as road segments connecting two junctions (alternatively called nodes, intersections, or vertices) develop and degenerate?

(2) How are new links added to the existing network?

(3) How are new nodes added to the existing network?

This paper addresses the first question and focuses only on the rise and fall of existing roads, recognizing the inter-dependence of road supply and travel demand. The approach is microscopic in that network dynamics are modeled at the link level. The following key questions are examined:

(1) Why do links expand and contract?

(2) Do networks self-organize into hierarchical patterns?

(3) Are roads (routes) an emergent property of networks?

(4) What are the parameters to be calibrated in a microscopic network dynamics model?

(5) Is the model computationally feasible on a realistic transportation network?

(6) Is the model capable of replicating real-world network dynamics?

Several previous studies (Weidner 1996; Horner and O'Kelly 2001; Yerra and Levinson 2005; Scott et al. 2006; Zhang and Levinson 2008; Miyagawa 2011) in this area show that transportation networks (road, air, etc.) tend to self-organize into a hierarchical pattern in which some links attract more trafficl users, receive proper maintenance, and are gradually expanded, while other links are less popular, poorly maintained, and may eventually be abandoned. It is also demonstrated that although this hierarchical pattern seems to be designed by planners and engineers, it is actually an intrinsic emergent property of networks themselves. The simulation model developed in Yerra and Levinson (2005) assumes unlimited road capacity; the growth and decline of roads are reflected only by changes in their free-flow speeds.

This paper makes several main contributions. First, travel demand is represented by a more realistic evolutionary demand pattern (extending Levinson [1995]) into the application of network growth). Second, it applies a routing and investment model akin to that of Zhang and Levinson (2009), thereby considering congestion effects and relaxing the assumption of unlimited capacity so that the impacts of network congestion on travel demand and supply can be incorporated in the analysis but applies it to a large-scale realistic network. Use of the Minneapolis-St. Paul, Minnesota (Twin Cities), transportation network with nearly 8000 nodes and more than 20,000 links allows us to examine computational properties and predictive value of the proposed microscopic network dynamics model, and it is the largest application to date of network evolution models. (Levinson, Xie, and Montes de Oca [2012] uses a similarly sized Twin Cities network, but only state-owned links in the choice set were eligible for possible expansion, and the investment rules were very different; see also Yusufzyanova and Zhang [2011b] for a similar analysis of the Washington, DC, and Maryland region.) 
The next section develops a theoretical framework for studying the rise and fall of roads. The framework helps identify various influencing factors and inter-dependences among those factors, based on which a synthesis model of road expansion and contraction is developed in Section 3. The model is applied to the Twin Cities transportation network from 1978 to 1998 with different model parameters and starting conditions. The results of these experiments are summarized, and the listed research questions are answered in Section 4. Conclusions and future research directions are offered at the end of the paper.

\section{Network dynamics at the microscopic level}

Regional economic growth is taken as exogenous for this study of transportation network dynamics because transportation infrastructure is not the only factor that drives economic growth, and we do not yet have adequate other models to explain change in land use at the metropolitan level. Some attempts at this include Levinson, Xie, and Zhu (2007). It has long been known that transportation service and land use influence each other though iterative changes in accessibility and travel demand. However, land-use dynamics are also treated as exogenous in the following network analysis, so that attention can be focused on transportation network growth, a process with enough complicated and unknown dynamics for one to start with. The dynamics of other factors involved such as travel behavior, link maintenance and expansion costs, network revenue, investment rules, link expansion and degeneration are considered endogenously.

The foremost and probably also the most important constraint on future network growth is the existing network. In developed countries where transportation infrastructure has reached its saturated stage, it is rare to see new network growth from a tabula rasa. Even in an empty place without any previous development, natural barriers such as rivers and mountains still constrain future network growth. The current network connectivity pattern determines whether two links complement (are located upstream or downstream) or compete with (are parallel to) each other for demand. The existing network may or may not be at a steady state. It could still take years for road supply to meet existing travel demand even if no exogenous changes (e.g., population and economic growth) occur. The important question is how various forces drive the existing network to evolve rather than how long it takes.

Based on the current network, land-use arrangements, and individual socioeconomic status, people make their travel decisions, such as trip frequency, scheduling, destination, mode and route choices. These decisions transform into travel demand on the transportation network. This demand-generating process involves the existing network supply, congestion externalities, travel behavior, and link-level travel demand forecasting.

Transportation is a service, and travelers pay to obtain that service in addition to spending their own travel time. In the United States, that payment is largely in the form of a fuel tax. However, if links were autonomous, they would set prices to maximize their profits in the form of a vehicle toll. In many real-world transportation networks, government agencies collect transportation revenue in terms of fuel taxes. We can set the price for using a link as a function of the link's length and speed. It is convenient to use a notion of link revenue. Revenues collected by individual links may or may not be pooled together for investment purposes depending on the underlying institutional structure of the network. Longer, faster, and high-demand (traffic flow) links should be able to generate more revenue. If not maintained appropriately, link speed will decrease over time due to physical deterioration caused by the environment and traffic. Therefore, each link has a maintenance cost function. Link length, capacity, free-flow speed, and flow determine maintenance cost to a large extent. The amount of money required to expand an existing link can be calculated with a link expansion cost function. A previous empirical estimate of link expansion costs using network data in the Twin Cities metro area reveals that link expansion cost is 
positively correlated to capacity, length, and road hierarchy (interstate, state highway, county highway, etc.), while negatively related to the distance from the nearest downtown (Levinson and Karamalaputi 2003). These results suggest that link length and capacity should be included in the link expansion cost function, and this function is also subject to local adjustments.

Specific revenue and cost structures in a transportation network provide inputs for investment decisions. Real-world observation of road investment decision processes suggests the hypothesis that decisions to expand transportation networks are largely myopic in both time and space, usually ignoring non-immediate and non-local effects (e.g., Montes de Oca and Levinson 2006; Yusufzyanova and Zhang 2011a; Zia and Koliba 2015). This myopic decision process, when applied sequentially, tends to improve the relative speeds and capacity of links that are already the most widely used, and thereby expand their use. The rate and extent of this process is constrained by the cost of those improvements and limited budgets (revenue). From a market economy point of view, travel demand increases (due to changing preferences as well as sheer population) induce supply (capacity) increases—suppliers produce more of a good. The output of the investment process would be an updated network where some links are expanded and some degenerated.

The mirror problem of induced demand is by now well understood (e.g., Noland 1998; Strathman et al. 2000; Fulton et al. 2000; Parthasarathi, Levinson, and Karamalaputi 2003). As travel costs drop, the number and especially the length of trips increase. The expanded link with increased travel demand can generate even more revenue, which may later result in further expansion on that link. Yet this loop, while positive, should have limits. The diminishing returns in the revenue structure and exponential increases of expansion costs will eventually stop this feedback loop. The opposite is true for degenerated links. All these suggest that reinforcement exists and transportation networks may self-organize into hierarchies. This hypothesis is subject to simulation tests in the following section.

Improving one link will also cause complementary (upstream and downstream) links to have greater demand, and competitors (parallel links) to have lesser demand (and be less likely to be improved). These network effects take time to propagate within transportation networks. They may be reinforced in complex transportation networks, create problems, but leave little clue to planners as to the root of the problem, and thus force transportation system planners and engineers to adopt myopic solutions, which may create even more problems.

This again highlights the importance of considering the full ramification of network expansion on future infrastructure decisions. Network effects both complicate the problem and suggest the analysis has to be iterative. Previous changes in the network, economy, demography, and even travel behavior cause a new travel demand pattern and hence new link costs and revenues. Accordingly, a new set of supply decisions will be made, generating new network changes. This loop is repeated until a steady state is achieved (depending on the cost structure, with fixed land-use patterns, the solution tends toward equilibrium). When the continuous exogenous changes in economy, technology, land development, and population are considered, a transportation network may never reach equilibrium. The evolutionary microscopic network growth process should produce rich dynamics important to anyone who is interested in shaping the future transportation network.

\section{A network dynamics model}

In this research, a network dynamics model is developed that brings together all the relevant agents and their interactions to simulate link expansion and contraction. Compared to an earlier network dynamics model due to Yerra and Levinson (2005), this improved model relaxes the assumption of unlimited link capacity, a necessary step that has to be taken to make the model be of any practical importance. The foundation for the model development is the microscopic network growth dynamics described in 
the previous section. The simulation model can be used to evaluate whether important system properties such as hierarchy, self-organization, and growth, actually emerge from decentralized processes. This purpose makes the principles of and modeling techniques for complex systems applicable. There is no universally accepted definition of a complex system. However, it is generally agreed that it consists of "a large number of components or 'agents,' interacting in some way such that their collective behavior is not a simple combination of their individual behavior" (Newman 2001), which is the case in transportation networks. Examples of complex systems include the economy-agents are competing firms; cities—places are agents; traffic—vehicles are agents; ecology—species are agents. In transportation networks, we model nodes, links, travelers, and land-use cells as agents. Cellular automata (CA) and agent-based modeling techniques are commonly employed tools for modeling complex systems (von Neumann 1966; Schelling 1969; Wolfram 1994, 2002). An agent-based structure is used in the proposed network dynamics model.

An overview of model components and their interconnectivity is shown in Figure 1. A travel demand model predicts link-level flows based on the network, socioeconomic, and demographic information. Based on the demand forecasting results, links calculate revenues and costs. An investment module then operates and causes annual supply changes, producing an updated network. The modeling process does not have to iterate annually. Other updating intervals can also be used. But yearly supply changes correspond to budgets, which are typically decided each fiscal year. The transportation network is represented as a directed graph that connects nodes with directional arcs (links). The standard notation convention for directed graphs is adopted for the following presentation on the details of mathematical formulations of those submodels. The directed graph is defined as: $G=\{N, A\}$ where $N$ is a set of sequentially numbered nodes and $A$ is a set of sequentially numbered directed arcs (links).

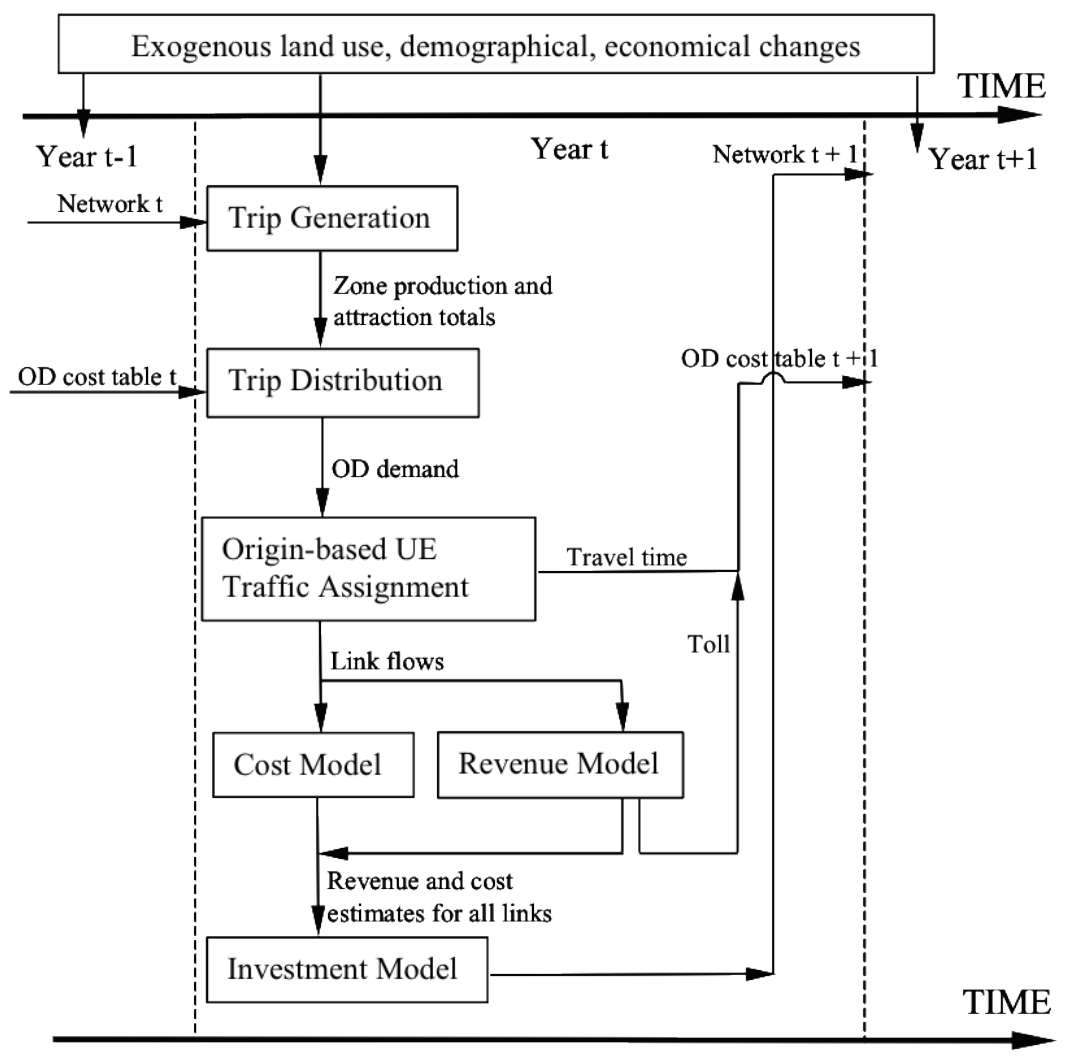

Figure 1: Flowchart of the transportation network dynamics model 


\subsection{Travel demand}

Ideally, an agent-based travel demand model in which node, link, and travelers are modeled as interactive agents should be applied to estimate travel demand at the level of links, so as to keep the disaggregate model structure consistent. Previous studies (Zhang and Levinson 2005, 2006, 2009; Zhang, Zhu, and Levinson 2008) have proposed such a model with successful application to the Chicago sketch network.

In this paper, a traditional four-step forecasting model is used to predict travel demand at the link level, taking exogenous land use, socio-economic variables, and the existing network as inputs, consistent with much current practice.

A zone-based regression structure is used for trip generation. The origin-destination (OD) cost table obtained from the previous-year traffic assignment is used for trip distribution in the current year based on a doubly constrained gravity model (Voorhees 2013; Wilson 1969; Haynes and Fotheringham 1984; Hutchinson 1974).

The computation of the new OD demand table takes into account the historical impacts of past travel behavior. Travel demand in a given year depends on the demand in the previous year. Levinson (1995) elaborates the idea of such a hybrid evolutionary model. In contrast to a traditional equilibrium model, the evolutionary demand updating procedure does not require supply and demand to be solved simultaneously. In this study, the new OD demand is updated by a process similar to the method of successive averages (MSA) (Sheffi 1985; Smock 1962) in traditional traffic assignment procedures. The weights in equation (1) are specified in such a way that OD demand tables in each preceding year are weighted equally with the current year (i) OD demand.

$$
q_{r s}^{i}=\left(1-\frac{1}{i}\right) q_{r s}^{i-1}+\left(\frac{1}{i}\right) m_{r} O_{r} n_{s} D_{s} \cdot d\left(t_{r s}^{i}\right)
$$

where:

$q_{r s}{ }^{i} \quad$ demand from origin zone $r$ to destination zone $s$ in year $i$

$O_{r} \quad$ number of trips produced from zone $r$

$D_{s} \quad$ number of trips destined for zone $s$

$m_{r}, n_{s} \quad$ coefficients in the gravity model

$t_{r s}^{i} \quad$ generalized travel cost of traveling from zone $r$ to $s$

$d\left(\right.$.) travel cost impedance function in the gravity model; $d\left(t_{r s}^{i}\right)=e^{-\gamma \cdot t_{r s}^{i}}$

$\gamma \quad$ coefficient in the impedance function

In the equation, the term $1 / i$ represents the fraction of total demand that comes from updating with the most recent estimate of travel times associated with the $i^{t h}$ year, while $1-1 / i$ represents the fraction of demand from all other years. This aims at recognizing that destination choices (i.e., home location vis$\grave{a}$-vis work location) are sticky and won't suddenly change with changes in travel times on the network.

In this model, all travelers are identical except for their origins and destinations, and thus have identical values of time. Other models have relaxed this assumption (e.g., Zhang, Zhu, and Levinson 2008) in different contexts.

The resulting OD table is loaded onto the current year transportation network through the originbased user equilibrium traffic assignment algorithm (OBA) developed by Bar-Gera and Boyce (2003). The generalized link cost function comprises two parts, a Bureau of Public Roads (BPR) (1964) travel time component and a vehicle toll. 


$$
t_{a}^{i}=\lambda \frac{l_{a}}{v_{a}^{i}}\left[1+\theta_{1}\left(f_{a}^{i} / F_{a}^{i}\right)^{\theta_{2}}\right]+\tau_{a}^{i}
$$

where:

$t_{a}^{i} \quad$ generalized travel cost on link $a$ in year $i$

$\lambda \quad$ value of travel time constant (dollar/hr)

$v_{a}^{i} \quad$ free-flow speed of link $a(\mathrm{~km} / \mathrm{hr})$ in year $i$

$F_{a}^{i} \quad$ capacity of link $a$ in year $i(\mathrm{veh} / \mathrm{hr})$

$l \quad$ the length of link $a$ (constant) $(\mathrm{km})$

$f_{a}^{i} \quad$ average peak hour flow on link $a$ in year $i(\mathrm{veh} / \mathrm{hr})$

$\theta_{1}, \theta_{2}$ coefficients of the BPR travel time function

$\tau_{a}^{i} \quad$ link toll per vehicle (dollar, see equation 4 for details)

The OBA algorithm derives link flows at user equilibrium and generates a new OD cost table that will be used for trip distribution in the next year. In the traffic assignment step, if the relative excess travel cost is less than 0.001, the Wardrop user equilibrium (Wardrop 1952) is considered to be satisfied. In practice, it is unlikely that traffic is actually at equilibrium (Zhu and Levinson 2015); however, this is standard practice in models, and guarantees a unique set of link flows, but not paths, and allows solutions to be systematically compared with fewer confounds.

\subsection{Revenue and cost}

Revenue is collected at the link level by vehicle toll. The annual revenue is simply the product of the toll and annual flow. The amount of the toll should depend positively on the length of the link and the level of service. In practice, toll roads often charge more for longer trips, which is not necessarily linear and depends on the system, and many also have a fixed cost or component of the toll that is independent of distance in addition to a variable (distance-based) part - though we assume it all to be linear and distance-based here. Also in practice, high occupancy/toll (HOT) lanes charge tolls for relatively faster trips compared with parallel free lanes, though tolling levels vary, and dynamic tolls rise with congestion (Janson and Levinson 2014). There is no standard guidance as to how much tolls would or should vary with speed. MnPASS, the local HOT lane, has a toll formula where the level of toll increases with density (and thus decreases in speed) in the HOT lane to ensure it remains free-flowing. In general, density increases in the HOT lane when the density also increases in the un-tolled and parallel general-purpose lanes, and thus travel-time savings are greater.

The following revenue equation is employed, adapting Yerra and Levinson (2005):

$$
\begin{aligned}
& E_{a}^{i}=\tau_{a}^{i} \cdot\left(\psi \cdot f_{a}^{i}\right) \\
& \tau_{a}^{i}=\rho_{1} \cdot\left(l_{a}\right)^{\rho_{2}} \cdot\left(v_{a}^{i}\right)^{\rho_{3}}
\end{aligned}
$$

where:

$E a^{i} \quad$ revenue (earnings) of link $a$ in year $i$ (dollar)

$\psi \quad$ coefficient to scale average peak hour flow to annual flow

$\rho_{1} \quad$ scale coefficient related to the toll level (dollar $\cdot \mathrm{hr}^{3} / \mathrm{km}^{2+\rho^{3}}$ )

$\rho_{2}, \rho_{3}$ coefficients indicating economies or diseconomies of scale 
As the free-flow speed of a link increases, travelers are able to save travel time and hence are willing to pay a higher toll. However, speed improvements have decreasing returns. For instance, if speed triples from 8 to $24 \mathrm{~km} /$ hour, time spent traveling $1 \mathrm{~km}$ drops 5 minutes from 7.5 minutes to 2.5 minutes. If speed increases $16 \mathrm{~km} /$ hour from $88 \mathrm{~km} /$ hour to $104 \mathrm{~km} /$ hour, the time drops from 41 seconds to 35 seconds — merely 6 seconds — which hardly seems worth considering.

The coefficient $\rho_{3}$ should be positive, because HOT lanes aside, tolls should not increase if speeds decrease, but less than 1 , because willingness to pay is unlikely to increase superlinearly with speed as time savings decrease with speed, as shown in the previous paragraph. Thus $\rho_{3}$ is between 0 and 1 . (We use 0.75 in the application below.) Note that with appropriate values for those coefficients, the tollbased link-level revenue structure can also reasonably model centralized revenue collection mechanisms, such as fuel taxes $\left(\rho_{2}=1\right.$ and $\left.\rho_{3}=0\right)$.

The link maintenance cost function has only two determining factors: Link length and capacity:

$$
C_{a}^{i}=\mu \cdot\left(l_{a}\right)^{\alpha_{1}}\left(F_{a}^{i}\right)^{\alpha_{2}}
$$

where

$C_{a}^{i} \quad$ cost of maintaining link $a$ at its present condition in year $i$ (dollar)

$\mu \quad$ scale parameter $\left(\right.$ dollar $\left.\cdot \mathrm{hr}^{\mathrm{a}} / \mathrm{km}^{\mathrm{a}}\right)$

$\alpha_{1}, \alpha_{2}$ coefficients indicating economies or diseconomies of scale

It is also assumed that all links have the same link maintenance cost function. This assumption is obviously not realistic and should be relaxed when local, link-specific data are available.

\subsection{Investment rules}

If a link is autonomous and its annual revenue is higher than maintenance cost, the link will be expanded in the next year, assuming revenue is not spent elsewhere. If revenue falls below maintenance cost, the link shrinks in terms of both a capacity reduction and a free-flow speed drop.

The submodel of network investment decisions can have two aims, describe reality or identify optimal policies. The emphasis in this paper is the prior one, which is in contrast to the long line of research on the network design problem. The network dynamics model must be able to replicate what has happened in reality before it is applied for potential planning purposes. A prototype investment rule (link expansion and contraction function) is examined in which links manage themselves and do not share revenues.

$$
F_{a}^{i+1}=F_{a}^{i}\left(E_{a}^{i} / C_{a}^{i}\right)^{\beta}
$$

where

$\beta \quad$ capacity change coefficient

Note that investment decisions in equation (6) are very myopic ones in that links only care about themselves, ignore network effects, and spend all revenues immediately. The value of $\beta$ actually represents some properties of the link expansion process. If $\beta$ is less than 1 , it implies that there are diseconomies of scale in link expansion because doubled investment $(E)$ would only produce less than doubled capacity. If $\beta$ is larger than 1 , economies of scale exist.

Link capacity changes are often correlated with free-flow speed. By standard highway design principles (e.g., the AASHTO [2011] "Green Book"), a hierarchy of roads has been designated where higher 
capacity roads are limited access and designed for higher speed. These design principles (which may have emerged in any case) have been widely employed throughout the United States. Free-flow speed and capacity data used by the Twin Cities Metropolitan Council in its regional transportation planning model on more than 10,000 roadway sections are used to study the correlation of speed and capacity. A $\log$-linear model is adopted (see Figure 2). $R^{2}$ of the model is 0.7 and both coefficients are statistically significant at level 0.01 .

$$
v_{a}^{i+1}=\omega_{1}+\omega_{2} \cdot \ln \left(F_{a}^{i+1}\right)
$$

The predicted free-flow speeds are plotted against data in Figure 2. Keeping component functions such as this one continuous and differentiable in the network dynamics model can save a lot of work in the calibration stage. This is also the reason why an explicit link expansion cost function is not specified and why it is assumed that links invest any extra revenue immediately. However, if these simple continuous functions cannot adequately replicate reality, more sophisticated modeling tools should be considered. For instance, link expansion and contraction are in fact discrete events for which a choice model or catastrophe theory may be applied.

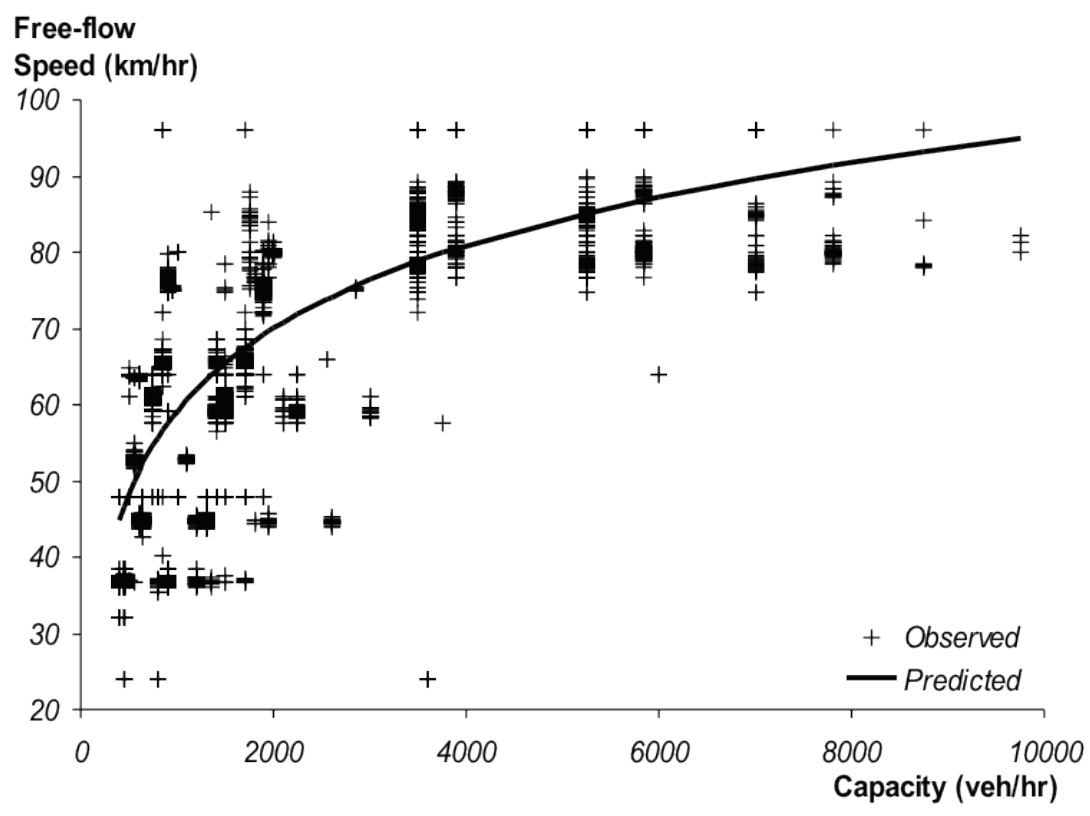

Figure 2: Link capacity and free-flow speed relationship: Observed (from regional transportation planning model) versus predicted (log-linear regression model)

With updated link capacity and free-flow speed, link travel time and link toll change, thus affecting travel behavior. These supply shifts combined with preference, economic growth, and demographic changes give rise to the emergence of a new demand pattern.

So far, a complete cycle of the network evolution process has been modeled. This cycle repeats itself annually. Simulation of these cycles can reveal various emergent properties of transportation network growth. The proposed network dynamics model can and should be calibrated and validated against observed time-series network and land-use data. The calibration procedure may consist of two stages. The parameters in the submodels (demand, revenue, cost, and investment) are estimated from empirical 
network data. These estimates then form a starting solution for an iterative optimization routine with an improving search algorithm. Finer adjustments to the model system and parameters should be undertaken based on an objective function, which can minimize the difference between the observed data and the model's ability to predict which links were improved and by how much. In brief, the model parameters form a space that can be searched systematically to find a best fit between actual and predicted link expansions and contractions. The transportation network data in the Twin Cities metro area have been collected between 1978 and the present in digital format, while data collection work on corresponding land-use and economic information is ongoing. In the 2000 Twin Cities transportation network, there were 7976 nodes and 20,914 links. A bit more than 600 link expansions took place between 1978 and 2000, which implies the Twin Cites transportation network is mature.

Though a rigorous calibration work cannot proceed unless all required data are collected, simulating the model with the available Twin Cities network data can still provide valuable information regarding the modeling concept, structure, and feasibility of a large-scale realistic network. The values of model parameters in these preliminary runs are based on either empirical estimation or our best understanding of the economies and diseconomies of scale in the network growth process and are summarized in Table 1. The simulation experiments also provide opportunities to examine some qualitative properties of network dynamics.

Table 1: Coefficients used in the experimental runs of the network dynamics model

\begin{tabular}{|c|c|c|c|}
\hline Parameter & Description & Value & Source \\
\hline$\lambda$ & value of travel time constant $(\$ / \mathrm{hr})$ & 10 & Empirical findings \\
\hline$\theta_{1}, \theta_{2}$ & coefficients in the BPR function & $0.15,4$ & $\mathrm{BPR}$ \\
\hline$\gamma$ & coefficient in the gravity model & 0.1 & Empirical findings \\
\hline$\rho_{1} \cdot \psi$ & 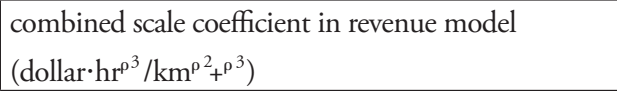 & 1 & Scale parameter \\
\hline$\rho_{2}$ & power term of length in revenue model & 1 & CRS of link length \\
\hline$\rho_{3}$ & power term of speed in revenue model & 0.75 & DRS of level of service \\
\hline$\mu$ & scale coefficient in cost model $\left(\right.$ dollar $\left.\cdot \mathrm{hr}^{\alpha 2} / \mathrm{km}^{\alpha 1}\right)$ & 20 & Scale parameter \\
\hline$\alpha_{1}$ & power term of length in cost model & 1 & CRS of link length \\
\hline$\alpha_{2}$ & power term of capacity in cost model & 1.25 & IRS of capacity \\
\hline$\omega_{1}, \omega_{2}$ & $\begin{array}{l}\text { coefficient in the speed-capacity log-linear regression } \\
\text { model }\end{array}$ & $-30.6,9.8$ & Empirical estimate based on Twin Cities data \\
\hline$\beta$ & capacity change coefficient & 0.75 & DRS in link expansion \\
\hline
\end{tabular}

CRS, DRS and IRS: constant, decreasing, and increasing returns to scale 


\section{$4 \quad$ Simulation experiments and results}

Four experiments are set up with different initial conditions and restrictions on link contraction. It is assumed in all experiments that there are no exogenous changes in land use, economy, and population based on 1998 Twin Cities Metropolitan Council data. Let us imagine that we are planners in 1978 who are interested in network growth 20 years from "now" (i.e., in 1998). The 1978 network thus becomes the "existing" network. So, in essence, these four experiments set up scenarios in which "estimated" land use 20 years from "now" is applied to the "existing" network. Using the real 1978 network as the initial condition for the simulation model (Experiments 1 and 2) allows us to observe whether and how this real-world network achieves steady state. The real 1978 network already exhibits hierarchy in that a few important roads carry the bulk of traffic, while most roads have relatively low speed and volume. To see how network hierarchy emerges in the growth path, the other initial condition is the 1978 network topology with a uniform capacity of 400 vehicles per hour, which is the capacity of the narrowest link in the 1978 network. The adoption of two initial scenarios can also reveal whether starting conditions significantly affect the future growth of a transportation network. In the investment model, link contraction occurs as long as the collected revenue is insufficient to maintain a link at its present condition. However, in reality, links usually do not shrink-once you build it, you cannot easily abandon it. The presence of this practical constraint is considered and applied to two of the four experiments (Experiments 2 and 4). Comparison of simulation results with and without the link contraction restriction sheds light on future refinement of the investment rules. In the simulation, if the network does not change in two consecutive years, the simulated network evolution process stops and a steady state is achieved. It is also possible that the network does not converge and changes constantly among two or more distinct states.

Table 2: Four simulation experiments

\begin{tabular}{|c|c|c|}
\hline \multirow{2}{*}{ Initial Condition } & \multicolumn{2}{|c|}{ Allow for Link Contraction } \\
\cline { 2 - 3 } & Yes & No \\
\hline 1978 Twin Cities network with real 1978 capacity & Experiment 1 & Experiment 2 \\
\hline 1978 network with uniform capacity $(400 \mathrm{veh} / \mathrm{h})$ & Experiment 3 & Experiment 4 \\
\hline
\end{tabular}

As we can see in Figure 3, thousands of links are expanded and contracted in the first several years following 1978. However, the network settles itself very quickly, and after about 25 years, fewer than 100 links still experience (relatively small) changes in capacity and free-flow speed. To achieve the strict equilibrium defined as a network with no more capacity changes, it is necessary to continue the iterations for many more years for any network as large as the one in the Twin Cities metropolitan area. But all significant changes occur during the first 20 years. It is clear the network dynamics model is approaching equilibrium smoothly. It is probably not practical to execute the model until a strict equilibrium is achieved. A goal function can be set up to determine the stopping point of the simulation. For instance, further iterations are not considered if the average percentage change of link capacity becomes less than 0.001 . The remaining presentation of the simulation results only focus on network dynamics between 1978 and 1998 since most important changes take place during this period. 


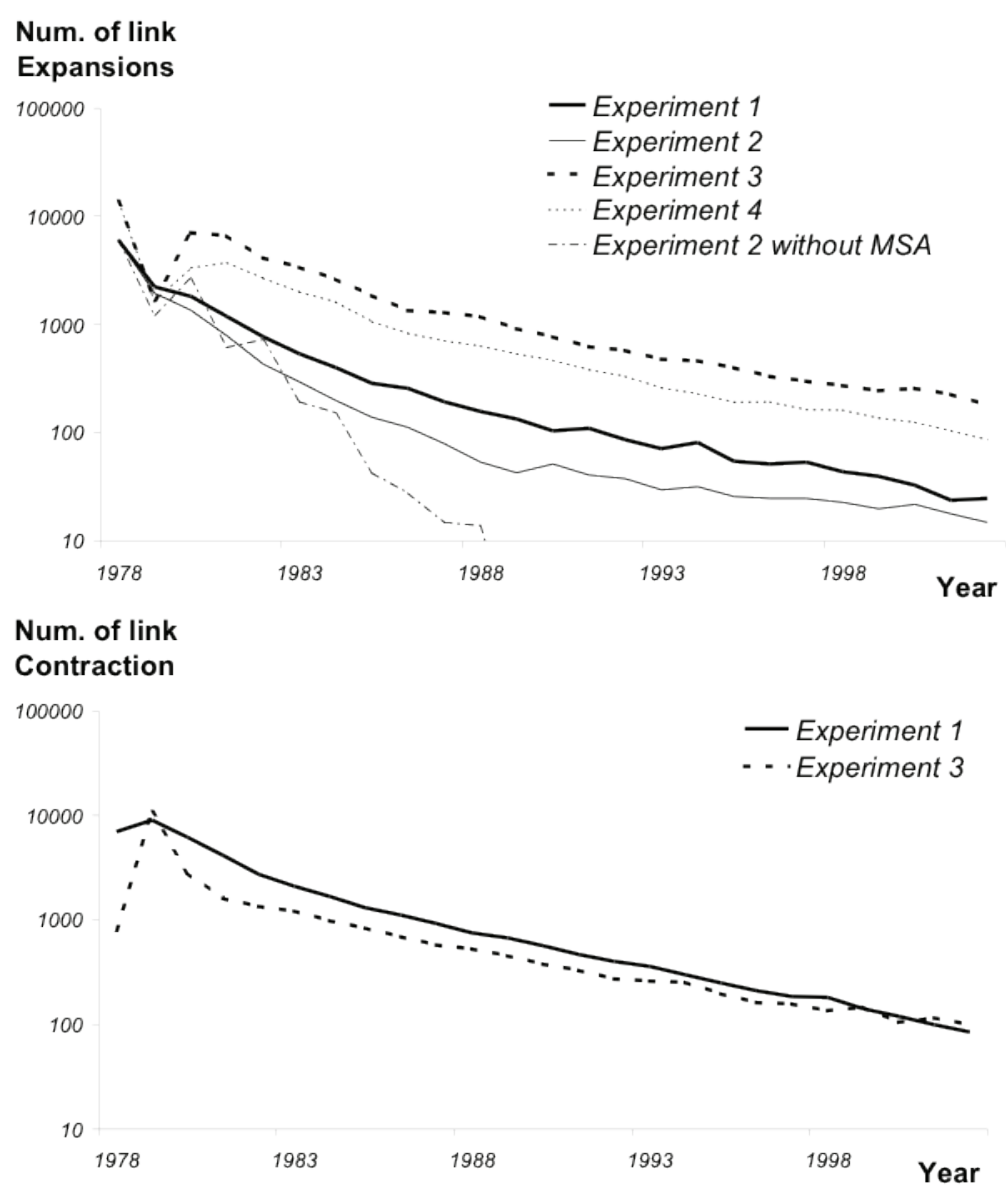

Figure 3: Convergence properties of the network dynamics model

In the travel demand model, an updating procedure similar to the method of successive averages (MSA) is adopted to take into account the impacts of lagged demand (Equation 1). One may suspect that it is this MSA procedure that forces the system to achieve a long-run equilibrium. To test this hypothesis, Experiment 2 with the fastest convergence is also executed without MSA, i.e., travel demand in the current year is independent of demand in previous years (only the second term on the right-hand side of Equation 1 remains in this case). The convergence property without MSA rejects the hypothesis and suggests induced or latent demand actually hinders the equilibration process of a transportation network. This result is also intuitive—a road system with factors delaying the adjustment of demand to changing supply, such as habitual behavior, uncertainty, and information acquisition cost, takes longer to reach its equilibrium than a system without those factors or with those factors to a lesser degree. However, the existence of lagged demand does not seem to affect the final state of the system (cf., Figures $4 d$, which is visually equivalent to the result without MSA (not shown)).

Predicted road expansions from the model between 1978 and 1998 are compared with expansion activities that actually occurred during that period. In Figure 4, the prediction results from Experiments $1(4 c)$ and $2(4 d)$ are compared to the observed data (4b). Although the model successfully predicts several large freeway construction projects, it forecasts more expansions on roads already having high capacities (freeway segments) and fewer expansions on arterial roads than reality. Either the expansion 
costs of arterial roads are overestimated or the costs of freeways are underestimated in the model. It is probably because the same cost function is used for all roads in the model. This suggests a need for cost functions adjusted to link-specific conditions. At this point, we are not arguing that the model predicts what should have been done. It must be able to describe reality first before it can be used as a normative tool.

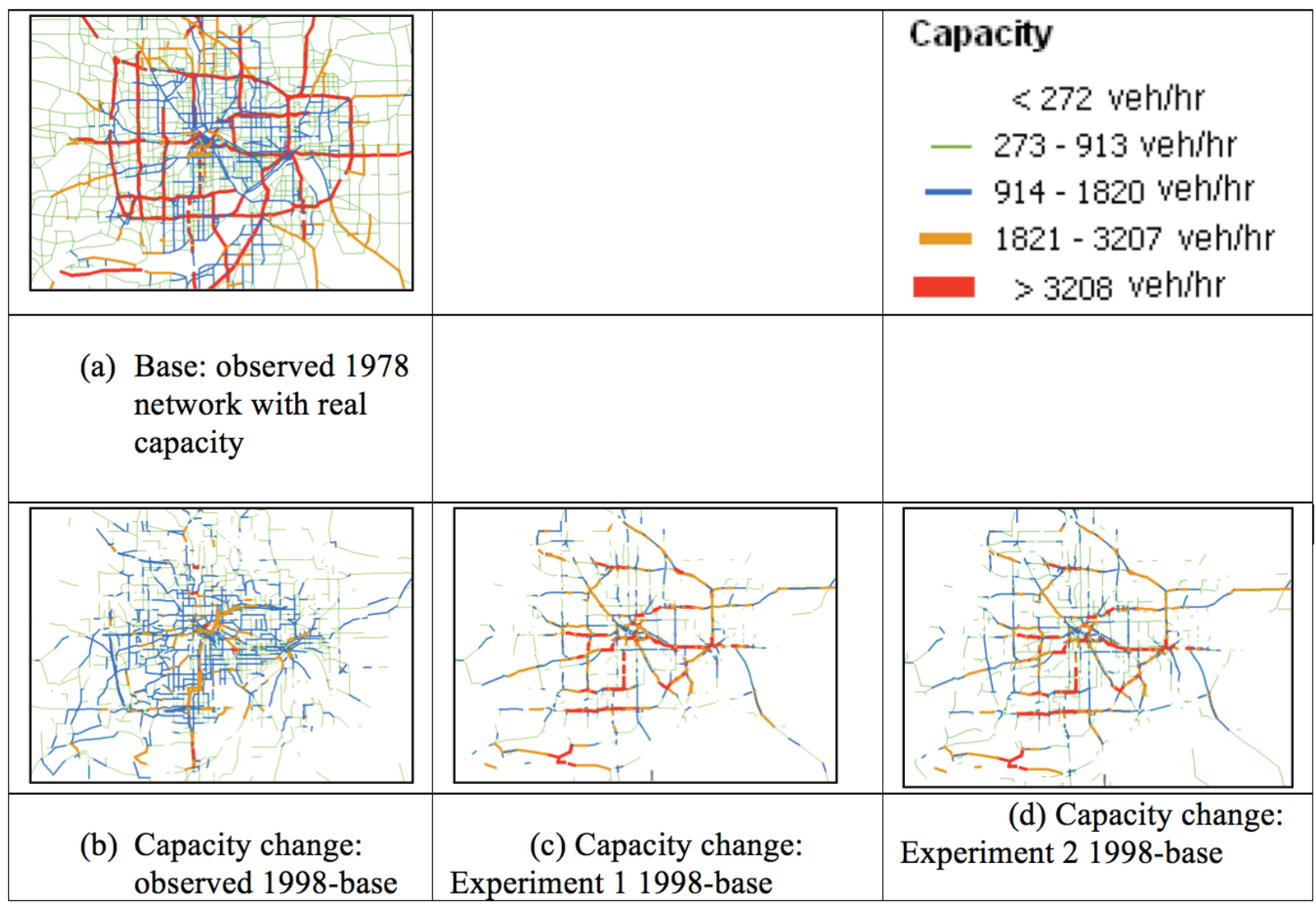

Figure 4: Experiment 1 and 2 vs. observed network growth after 20 years

Road hierarchy emerges in all four experiments (see Figure 5). In the predicted 1998 networks, most roads have low capacity and carry low flows, while only a few roads are expanded to very high capacities and carry the bulk of traffic. Experiments 1 and 2 start from the 1978 network with real capacity and hence the hierarchical structure is already present at the initial condition because the construction work of most freeways in the Twin Cites had been completed by 1978. It is, therefore, not very surprising to see that the predicted 1998 network hierarchy conforms very well with the observed 1998 data. With accurate network data in the starting year (1978) and good exogenous forecasts of land use and economic growth in a future year (we actually observed the 1998 land use), the proposed network dynamics model with very simple decentralized cost, revenue, and invest functions provides satisfactory forecasts of road hierarchy in the future year. It is interesting to see that hierarchy also emerges in Experiments 3 and 4, where the starting condition is a uniform capacity network. The predicted hierarchies in these two scenarios are actually very close to the observed ones for lower-level roads. The results from Experiments 3 and 4 also suggest that if planners in the Twin Cities could design a brand-new network to serve the existing travel demand and replace the existing network, they would build many fewer roads 
with very high capacities, as seen on the right side of the two graphs. This finding may be somehow not very meaningful due to the big "if." How the network arranges itself in a hierarchical pattern from a uniform status is a really interesting question. To answer that question, the growth path of the Twin Cities network in Experiment 4 is presented in consecutive maps, where changes in road capacity are shown with lines with different weights (Experiment 3 gives almost the same results and is therefore not shown).
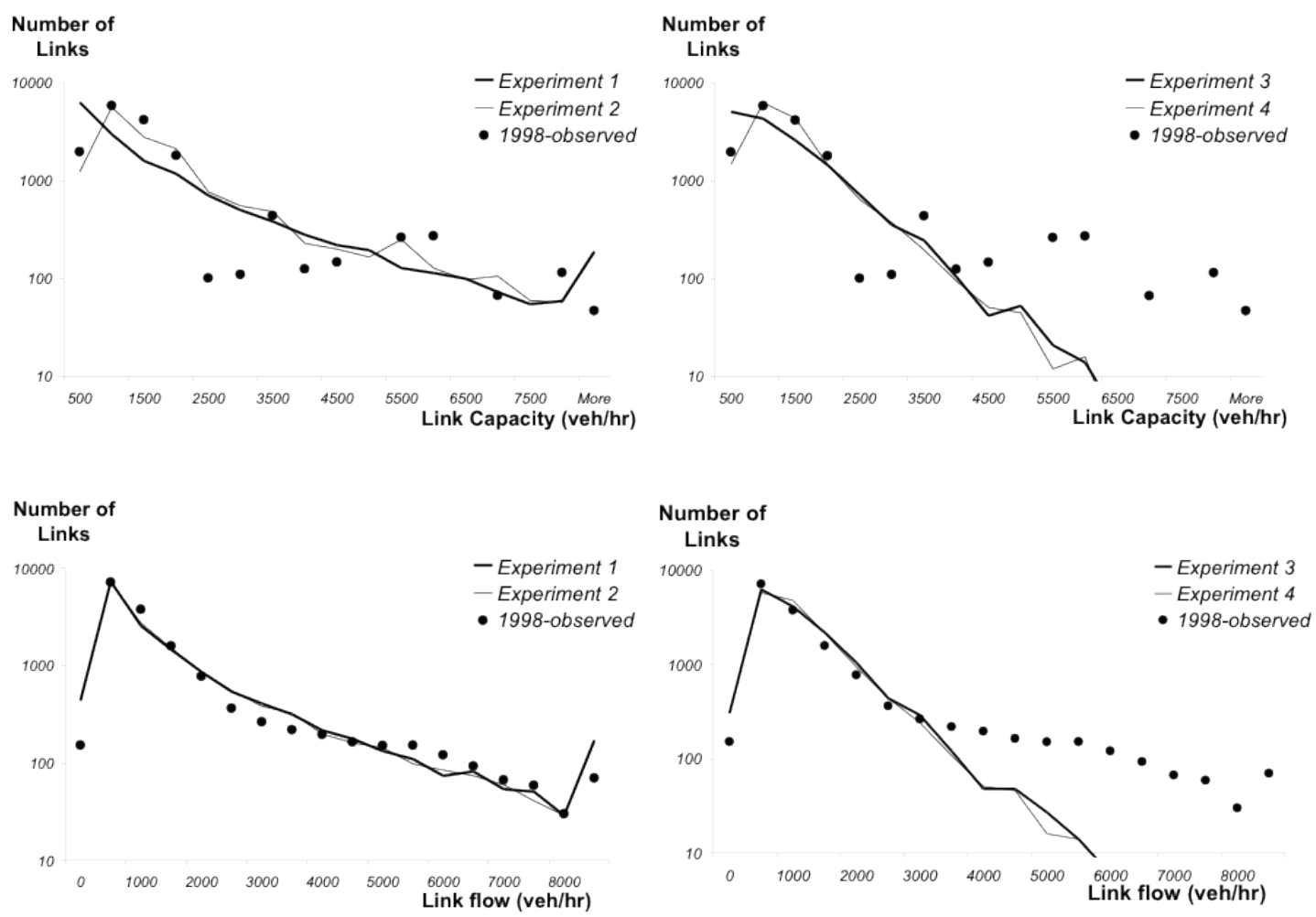

Figure 5: Road hierarchy after 20 years

For those who are not familiar with the Twin Cities metropolitan area, a brief description of the features of the region may be helpful before the maps in Figure 6 are examined. Two traditional central business districts, downtown Minneapolis and downtown Saint Paul, are approximately 10 kilometers from each other. At the confluence of the Minnesota and Mississippi Rivers is the region's international airport. A newer suburban business area, along the I-494 beltway, appears near the airport. The three downtowns, as well as the rivers are shown in the base year network (6a). After four years, the model predicts that some roads are expanded (6b). The location of these expansions tells us much about how road hierarchy emerges even from a uniform network. Natural barriers, such as rivers in this case, are sources of unbalanced road construction. It is clear that bridges are able to attract more flow than other roads in the network and hence are expanded first. Network effects then drive more flow to the roads emanating from bridges, for instance, the roads along riverbanks. If one carefully examines the roads surrounding the airport, the circle just west of the river confluence, it is evident these roads are also able to generate more revenues than an average road and are expanded early in the evolution process. The role of the airport here is much like some natural barriers such as mountains, because they all direct more flow to bypasses. The second source of hierarchy comes from activity centers. The three downtowns with 
high densities of jobs and other activities are the areas with intense road expansions in the years following 1978. Finally, the fact that all major road expansions between 1978 and 1982 take place in the central area of the region suggest that boundary effects also contribute to the formation of road hierarchy. Most trips originating from the edges of the city are inward trips and destined for activity centers located relatively closer to the geographical center of the region, while trips emanating from areas in the middle of the city are distributed along all possible directions. The asymmetry in demand patterns is the third source of road hierarchy identifiable from the second map. Again, network effects will help propagate the hierarchical pattern created by those three sources throughout the whole network over time. Twenty years later, road hierarchy can be found virtually everywhere in the network (6c).

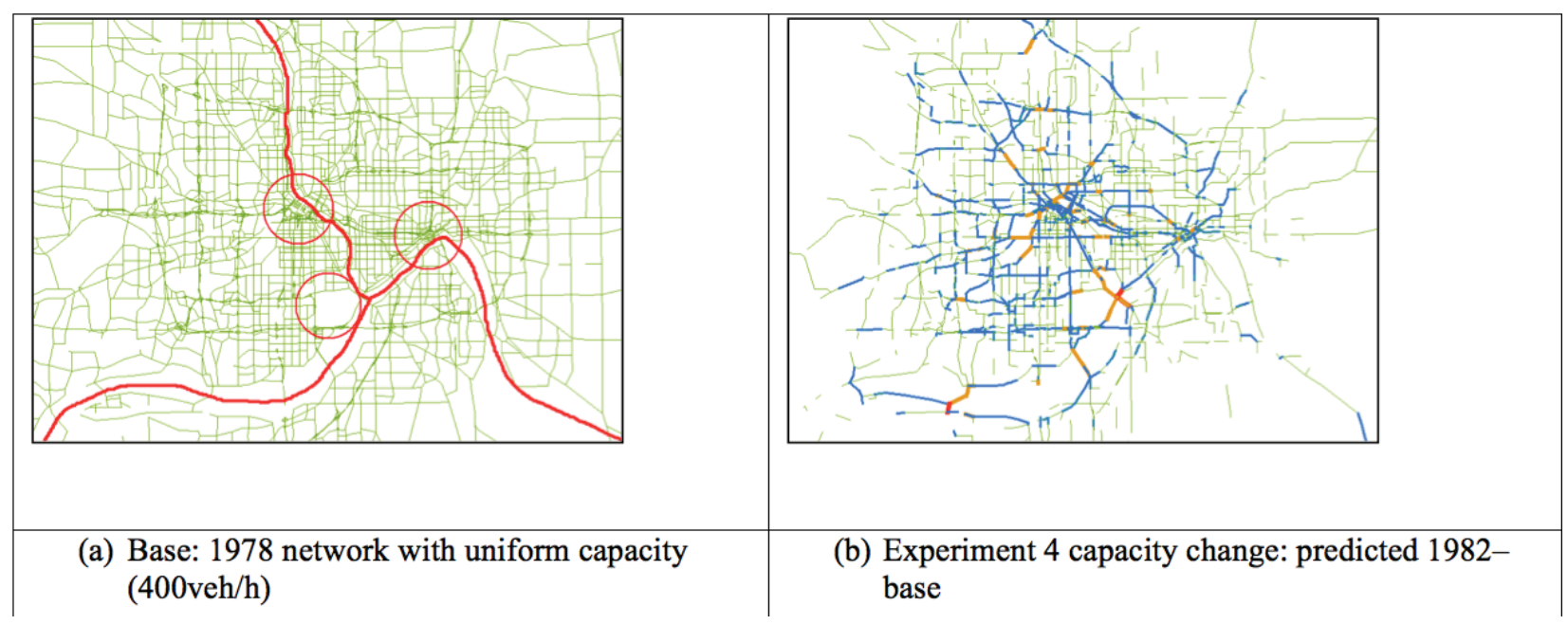

\begin{tabular}{|l|c|}
\hline & $\begin{array}{r}\text { Capacity } \\
<272 \text { vehihr } \\
-273-913 \text { vehihr } \\
-914-1820 \text { wehihr } \\
1821-3207 \text { vehihr } \\
>3208 \text { vehihr } \\
>\end{array}$ \\
\hline (c) Experiment 4 capacity change: Predicted & \\
1998-base &
\end{tabular}

Figure 6: Emergence of hierarchies in Experiment 4 
Congestion is undesirable in a network and attracts a lot of attention in network analysis. In Figure 7 , volume to capacity ratios (V/C ratios) of all roads in the network after 20 years of evolution are plotted in a histogram. The observed 1998 data suggest that most roads carry flows well below their capacity, and a few roads operate at $\mathrm{V} / \mathrm{C}$ ratios near or slightly higher than one. Practically, over a long period of time, no road can carry flows more than its capacity. The presence of $\mathrm{V} / \mathrm{C}$ ratios larger than one in the model is the result of inadequate description of road travel delays and scheduling adjustments in the traditional four-step, travel-forecasting model. Experiments 1 and 3 allow road degeneration, the results from which show a narrow range of $\mathrm{V} / \mathrm{C}$ ratios, suggesting a more uniform distribution of congestion in the network compared to the observed data. Note that the model does not say that at equilibrium a uniform distribution of $\mathrm{V} / \mathrm{C}$ ratios will be achieved. Roads have similar V/C ratios in Experiments 1 and 3 but not the same ratios. Experiments 2 and 4, with a constraint on road contraction, obviously predict congestion much better than their counterparts without the constraint. Once a road is expanded but demand later does not justify the capacity after the expansion, the road is still going to be maintained and capacity reduction is less likely to happen. Furthermore, in the real-world, capacity expansions are discrete (1 lane, 2 lanes), while here they are modeled as continuous. Therefore, a constraint on road degeneration in the model should make it more realistic. The spike near a $\mathrm{V} / \mathrm{C}$ ratio of one is still present in Experiments 2 and 4. This is because the same revenue and cost functions are applied to all roads. In reality, it may be more expensive to expand some roads than others and hence different levels of congestion are observed. This again suggests that cost and revenue functions in the model should be adjusted according to local conditions.

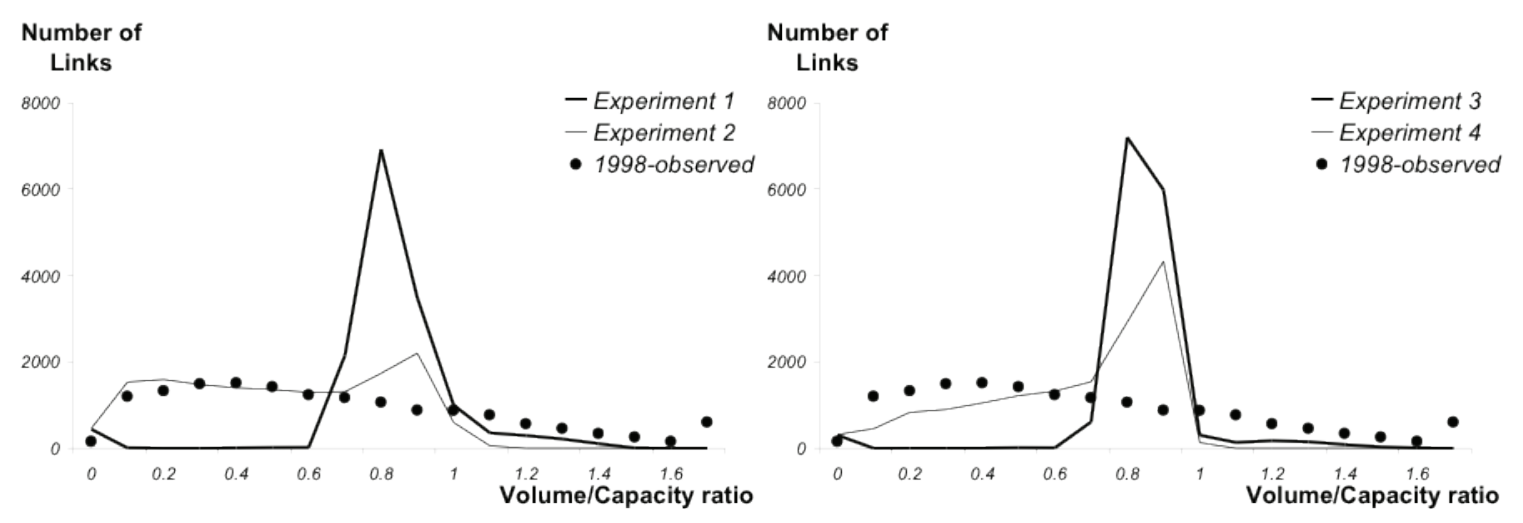

Figure 7: Network congestion after 20 years

Finally, the impacts of starting conditions and constraints on the predicted network dynamics are examined in Figure 8. Clearly, they do matter. By comparing the four graphs vertically, i.e., Experiment 1 against 3, and 2 against 4, we find that different initial networks result in quite different networks at equilibrium. A horizontal comparison of the graphs reveals the relatively smaller influence of the restriction on road degeneration. 


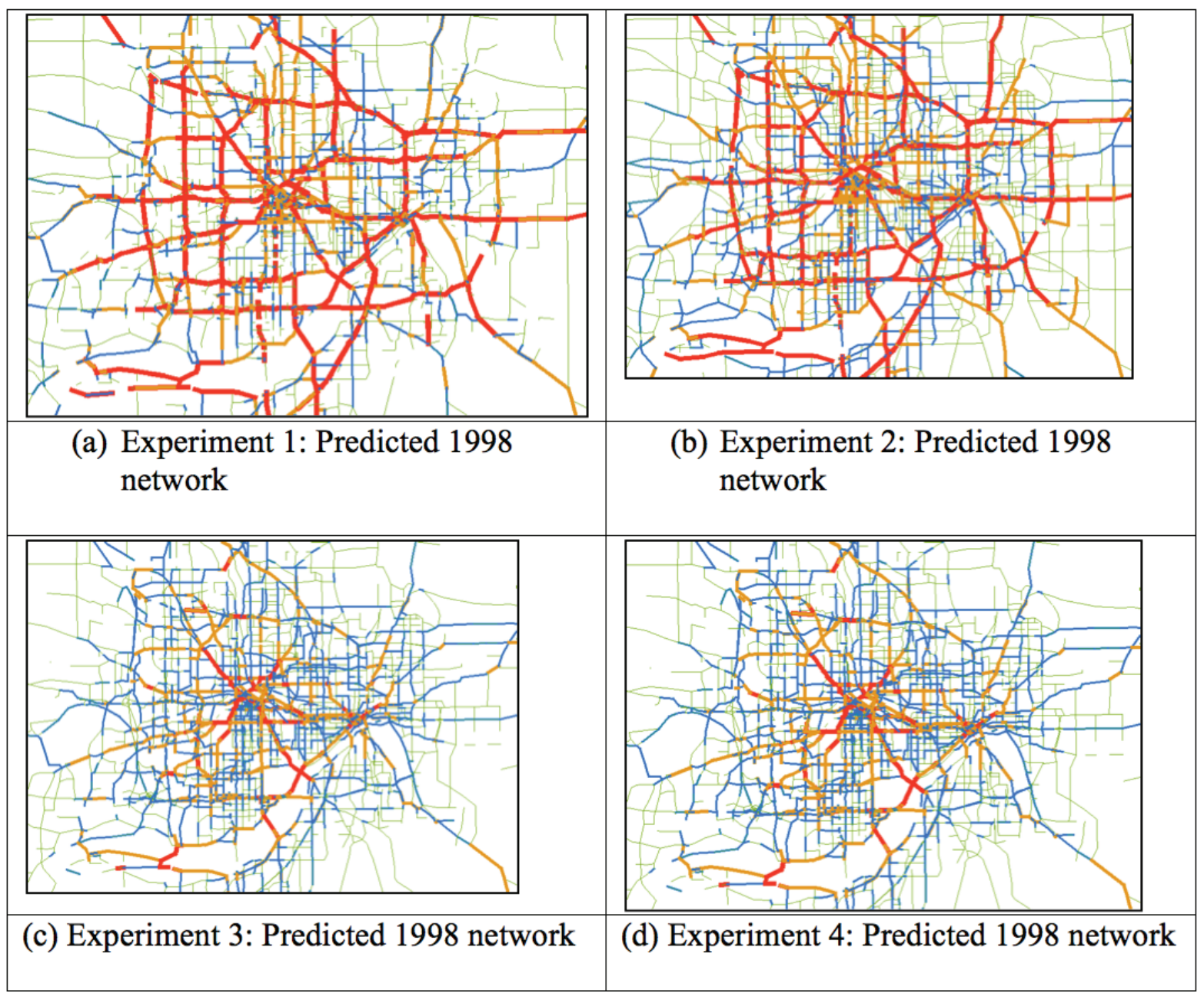

Figure 8: The impacts of starting conditions and constraints on network growth

\section{$5 \quad$ Conclusions}

A transportation network is a very complex system that consists of a full spectrum of various subsystems, the properties and behaviors of which are already hard to forecast. Efforts put into travel demand forecasting, network design problems, and revenue policies by numerous researchers are evidence of such difficulties. Predicting the growth of transportation networks is difficult because it requires us to consider almost all subprocesses involved in network dynamics. Understanding the true relationships between supply and demand in transportation networks is the crucial task in theoretical development of network dynamics models. The difficulty also comes from practical issues, such as available data for model calibration and validation. Socioeconomic, demographic, land-use, and transportation-network data from many years ago in an urban area must be collected and coded consistently over time. Several unresolved issues further complicate the problem, with the foremost one being: Is network growth simply designed by planners, or can it be explained by underlying natural and market forces? In light of this debate, we would like to view this paper as a proof of concept that some important system properties, such as road hierarchy and self-organization in transportation networks, can be predicted through a microscopic evo- 
lutionary process, a demonstration that such a microscopic agent-based model of network dynamics can be feasibly applied to large-scale realistic transportation networks, and an enquiry into how this concept can be realized and produce useful modeling tools for planners. Growth of economy, population, and cities has been intensively studied and knowledge accumulated from such studies has greatly aided planners. Traditionally, transportation networks have been assumed to be static or predetermined in analysis of urban areas. A model of transportation network dynamics can reveal more completely the impacts of today's planning decisions in the future.

The present paper explores only the rise and fall of existing roads (maybe the rise and rise of existing roads given the preliminary simulation results), leaving the questions of how new roads are built and new nodes are created in realistic transportation networks to be answered by other studies. The process of road development and degeneration at the microscopic level is analyzed and an agent-based simulation structure seems to be appropriate for modeling that process. To better describe reality, a systematic way to adjust cost and revenue functions based on area-specific factors such as type of roads, land value, and public acceptance should be considered. The inclusion of different classes of users with different values of time is an important extension. Similarly, the consideration of trucks (heavy duty vehicles), which have different operating characteristics and different cost structures associated with pavement wear and tear, would enhance the model. Another extension to the proposed network growth model is the incorporation of multiple travel modes, such as transit, rail, and pedestrian networks, which would allow planners to examine substitutional and complementary effects among various modes, and to evaluate a broader spectrum of transportation revenue and investment policies. Further research should test the sensitivity of models like this to parameters and consider constraints such as geometric design guidelines to better understand how constraints affect growth. 


\section{References}

AASHTO. 2011. A Policy on Geometric Design of Highways and Streets, 6th edition. Washington DC: American Association of State Highway and Transportation Officials.

Bar-Gera, H., and D. Boyce. 2003. Origin-based algorithm for combined travel forecasting models. Transportation Research 37B(5): 405-22.

Barthelemy, M. 2015. Time Evolution of Road Networks. InTraffic and Granular Flow'13, pp. 317337. Cham, Switzerland: Springer International Publishing.

Bureau of Public Roads. 1964. Traffic Assignment Manual. Washington, DC: US Department of Commerce.

Fulton, L. M., R. B. Noland, D. J. Meszler, and J. V. Thomas. 2000. A statistical analysis of the induced travel effects in the US Mid-Atlantic region. Journal of Transportation and Statistics 3(1): 1-14.

Haynes, K. E., and A. S. Fotheringham. 1984. Gravity and Spatial Interaction Models. Beverly Hills, CA: Sage Publications.

Horner, M. W., and M. E. O'Kelly. 2001. Embedding economies of scale concepts for hub network design. Journal of Transport Geography 9(4): 255-265.

Hutchinson, B. G. 1974. Principles of Urban Transportation Systems Planning. New York: McGraw-Hill.

Janson, M., and D. Levinson. 2014. HOT or not: Driver elasticity to price on the MnPASS HOT lanes. Research in Transportation Economics 44: 21-32.

Levinson, D. 1995. An evolutionary transportation planning model. Transportation Research Record 1493: 64-73.

Levinson, D., and R. Karamalaputi. 2003. Induced supply: A model of highway network expansion at the microscopic level. Journal of Transport Economics and Policy 37(3): 297-318.

Levinson, D., F. Xie, and S. Zhu. 2007. The co-evolution of land use and road networks. In Transportation and Traffic Theory 2007, edited by R. E. Allsop, M. G. H. Bell, and B. Heydecker, pp. 839-859. Bingley, UK: Emerald Group Pub.

Levinson, D., F. Xie, and N. Montes de Oca. 2012. Forecasting and evaluating network growth. Networks and Spatial Economics 12(2): 239-262.

Miyagawa, M. 2011. Hierarchical system of road networks with inward, outward, and through traffic. Journal of Transport Geography 19(4): 591-595.

Montes de Oca, N., and D. Levinson. 2006. Network expansion decision making in Minnesota's Twin Cities. Transportation Research Record 1981: 1-11.

Newman, M. E. J. 2001. The structure and function of networks. Computer Physics and Communications 147: 40-45.

Noland, R. B. 1998. Relationship between highway capacity and induced vehicle travel. Transportation Research Board 78th Annual Meeting Preprint CD-ROM. Washington, DC: Transportation Research Board, National Research Council.

Parthasarathi, P., D. Levinson, and R. Karamalaputi. 2003. Induced demand: A microscopic perspective. Urban Studies 40(7): 1335-1353.

Schelling, T. C. 1969. Models of segregation. American Economic Review 59(2): 488-93.

Scott, D. M., D. C. Novak, L. Aultman-Hall, and F. Guo. 2006. Network robustness index: A new method for identifying critical links and evaluating the performance of transportation networks. Journal of Transport Geography 14(3): 215-227.

Sheffi, Y. 1985. Urban Transportation Networks. Englewood Cliffs, NJ: Prentice-Hall.

Smock, R. J. 1962. An iterative assignment approach to capacity restraint on arterial networks. Highway Research Board Bulletin 156: 1-13. 
Strathman, J. G., K. J. Dueker, T. Sanchez, J. Zhang, and A. E. Riis. 2000. Analysis of induced travel in the 1995 NPTS. Portland, OR: Center for Urban Studies, Portland State University.

Von Neumann, J. 1966. Theory of Self Reproducing Automata, edited by A. W. Burks. Champaign, IL: University of Illinois Press.

Voorhees, A. M. 2013. A general theory of traffic movement. Transportation 40(6): 1105. (Republished from Institute of Transportation Engineers 1955).

Wardrop, J. G. 1952. Some theoretical aspects of road traffic research. Proceedings of the Institution of Civil Engineers, Part II 1(36): 325-62.

Weidner, T. 1996. Hubbing in US air transportation system: Economic approach. Transportation Research Record (1562): 28-37.

Wilson, A. G. 1969. The use of entropy maximizing models, in the theory of trip distribution, mode split and route split. Journal of Transport Economics and Policy 3: 108-126.

Wolfram, S. 1994 Cellular Automata and Complexity. Boston, MA: Addison-Wesley.

Wolfram, S. 2002. A New Kind of Science. Champaign, IL: Wolfram Media.

Xie, F., and D. Levinson. 2009. Modeling the growth of transportation networks: A comprehensive review. Networks and Spatial Economics 9(3): 291-307.

Yerra, B., and D. Levinson. 2005. The emergence of hierarchy in transportation networks. Annals of Regional Science 39(3): 541-553.

Yusufzyanova, D., and L. Zhang. 2011a. Multi-modal and multi-jurisdictional transportation investment decision-making: The case of Washington, DC-Baltimore Region. In Transportation Research Board 90th Annual Meeting (No. 11-4157).

Yusufzyanova, D., and L. Zhang, L. 2011b. Forecasting transportation network evolution and performance under existing and alternative transportation planning processes. In 11 th International Conference of Chinese Transportation Professionals, 4145-4156.

Zhang, L., and D. Levinson. 2005. An agent-based approach to travel demand forecasting: An exploratory analysis. Journal of the Transportation Research Board 1898: 28-36.

Zhang, L., and D. Levinson. 2006. Road pricing with autonomous links. Journal of the Transportation Research Board 1932: 147-155.

Zhang, L., and D. Levinson. 2008. Investing for reliability and security in transportation networks. Journal of the Transportation Research Board 2041: 1-10.

Zhang, L., and D. Levinson. 2009. Economics of network ownership. International Journal of Sustainable Transportation 3(5): 339-359.

Zhang, L., S. Zhu, and D. Levinson. 2008. Agent-based modeling of price competition, capacity choice, and product differentiation in congested networks. Journal of Transport Economics and Policy 42(3): 435-461.

Zhu, S., and D. Levinson. (2015). Do people use the shortest path? An empirical test of Wardrop's first principle. PloS One, 10(8), e0134322.

$\mathrm{Zia}$, A., and C. Koliba. 2015. The emergence of attractors under multi-level institutional designs: Agentbased modeling of intergovernmental decision making for funding transportation projects. $A I$ and Society 30(3): 315-331. 